\title{
Técnica de lavagem do conduto auditivo para medir a infestação por Psoroptes cuniculi em caprinos
}

\author{
Washing the auditory canal to determine the infection by Psoroptes cuniculi in goats
}

\author{
Malba Gean Rodrigues de Amorim ${ }^{\mathrm{I}}$ Ana Célia Athayde ${ }^{\mathrm{I}}$ Ana Clara Gomes dos Santos ${ }^{\mathrm{II}}$ \\ Franklin Riet-Correa $^{{ }^{*}}$
}

\section{- NOTA -}

\section{RESUMO}

Esta pesquisa teve como objetivos avaliar a eficiência da técnica do jato de água, com 50 e 100ml de água, para determinar a prevalência da infecção por Psoroptes cuniculi e estimar o grau de infestação para estudos da dinâmica populacional. $O$ estudo foi realizado no matadouro municipal da cidade de Patos, Paraíba, com 30 caprinos de ambos os sexos e idades variadas. $O$ conduto auditivo esquerdo de cada caprino foi lavado com $100 \mathrm{ml}$ de água e o direito, com $50 \mathrm{ml}$. Os ácaros coletados na lavagem e os ácaros obtidos após a abertura dos condutos auditivos foram contados e identificados de acordo com o estádio do ciclo biológico do parasito. Mediante a técnica da lavagem com 100mL, foi identificado o parasita em 22 (91\%) dos 24 condutos infectados e, na lavagem com $50 \mathrm{~mL}$, foi identificado em $18(78,3 \%)$ de 23 condutos infectados $(P>0,05)$. Após a abertura e contagem do total de ácaros, verificou-se que, na lavagem, haviam sido recuperados de 19\% a 35\% dos ácaros de todos os estádios de desenvolvimento, exceto para o estádio de ovo, que foi de $11 \%$ a 15\%, não havendo diferença estatística significativa $(P>0,05)$ entre as taxas de recuperação com 50 e $100 \mathrm{~mL}$ de água. Concluise que a técnica da lavagem do conduto auditivo é eficiente para estudos da prevalência da otocariase, porém, para estudos de dinâmica populacional, recomenda-se a abertura dos condutos auditivos e a contagem de todos os ácaros presentes.

Palavras-chave: caprinos, estudos epidemiológicos, Psoroptes cuniculi, sarna soróptica.

\section{ABSTRACT}

The aim of this study was to evaluate the technique of washing the auditory canal (ear flushing technique) either with 50 or $100 \mathrm{~mL}$ of water to determine the prevalence of the infection by Psoroptes cuniculi and to estimate the degree of infestation for population dynamics studies. The experiment was conducted in a slaughterhouse in the city of Patos, state of Paraiba, northeastern Brazil, with 30 goats of different ages and both sexes. Left auditory canal of each goat was washed with $100 \mathrm{~mL}$ of water, and the right with $50 \mathrm{~mL}$. Later, the auditory canals were opened to count the remaining parasites. The mites recovered by washing and those recovered after opening the auditory canals were counted and identified in accordance with the stage of development. No significant differences $(P=5.81)$ were found in the prevalence of the infection between the auditory canals washed with $50 \mathrm{~mL}$ (78.3\%), and those washed with $100 \mathrm{~mL}$ (91\%). After opening the auditory canals it was determined that the parasites recovered by washing represented $19 \%$ to $35 \%$ of the total parasites of the auditory canal for all stages of development, except for the eggs, which varied from $11 \%$ to $15 \%$ of recovering, with no significant differences $(P>0,05)$ between the auditory canals washed with 50 or $100 \mathrm{~mL}$. It is concluded that the technique of washing the auditory canals with 50 or $100 \mathrm{~mL}$ of water in goats is efficient to study the prevalence of infection by $\boldsymbol{P}$. cuniculi, however the intensity of the infection and the population dynamics should be studied in slaughtered goats by opening the auditory canals to count the different stages of the parasite.

Key words: epidemiologic studies, goats, Psoroptes cuniculi, psoroptic mange.

A sarna psoróptica dos caprinos, causada por Psoroptes cuniculi, localiza-se no conduto auditivo externo. No Brasil, P. cuniculi já foi encontrado parasitando caprinos em vários estados incluindo São Paulo (ARAÚJO, 1941), Rio de Janeiro e Pernambuco (FACCINI et al., 1981), Ceará (COSTA \& VIEIRA, 1984), Paraíba, Acre e Minas Gerias (FACCINI \& COSTA, 1992). Dentre as técnicas utilizadas nos estudos epidemiológicos de $\boldsymbol{P}$. cuniculi, encontram-se a técnica de necropsia em animais de abatedouros, o exame direto mediante

\footnotetext{
'Hospital Veterinário, Universidade Federal de Campina Grande (UFCG), 58700-000, Patos, PB, Brasil. E-mail: franklin.riet@pq.cnpq.br. *Autor para correspondência.

IIṔs-graduação em Ciência Animal, Universidade Estadual do Maranhão (Uema), São Luís, MA, Brasil. Recebido 11.11.13 Aprovado 21.02.14 Devolvido pelo autor 05.06.14 CR-2013-1512.R1
} 
swab e o lavado dos condutos auditivos dos animais com 50ml de água (FACCINI \& COSTA, 1992).

Esta pesquisa teve como objetivos avaliar a eficiência da técnica do jato com 50 ou $100 \mathrm{ml}$ de água para determinar a prevalência da infecção por P. cuniculi em caprinos e a eficiência da técnica em estimar o grau de infestação para estudos da dinâmica populacional.

O estudo foi realizado no matadouro municipal da cidade de Patos, localizado no sertão da Paraíba (S $07^{\circ} 01^{\prime} 28^{\prime \prime}$; W $37^{\circ} 16^{\prime} 48^{\prime}$ "), em 30 caprinos abatidos, sem raça definida, de ambos os sexos e idades variadas. O conduto auditivo esquerdo de cada caprino foi lavado com $100 \mathrm{~mL}$ de água e o direito com $50 \mathrm{~mL}$, de acordo com a técnica do jato de água, que consiste na lavagem do conduto auditivo externo com um jato forte de água, capaz de desprender todo o cerume e outras secreções juntamente com os ácaros existentes (LEITE et al., 1989). Após a lavagem, os condutos auditivos dos animais foram obstruídos com chumaço de algodão, para impedir a saída dos ácaros, e as cabeças dos animais foram separadas do corpo, acondicionadas individualmente em sacos plásticos e transportadas para o laboratório de Patologia Animal do Hospital Veterinário da Universidade Federal de Campina Grande. No laboratório, a contagem dos ácaros restantes foi realizada após a abertura dos condutos auditivos (NUNES \& NUNES, 1975). Os ácaros coletados na lavagem e os ácaros obtidos após a abertura dos condutos auditivos foram acondicionados, separadamente, em vidros contendo álcool a 70\%, contados e identificados de acordo com o estádio do ciclo biológico do parasito, utilizando a chave taxonômica descrita por SWEATMAN (1958).

Para verificar a eficiência da técnica em recuperar ácaros nos diferentes estádios de $\boldsymbol{P}$. cuniculi, foi usada a seguinte fórmula: [AL/(Al+AR) ] x $100 \mathrm{em}$ que $\mathrm{AL}=$ ácaros contados na lavagem e $\mathrm{AR}=$ ácaros contados após a abertura dos condutos auditivos. Os índices parasitários utilizados para estimar o tamanho das populações de parasitos foram: densidade de ácaros no conduto auditivo, intensidade média de infestação, variação da intensidade e abundância média (MARGOLIS et al., 1982).

Para comparar a eficiência da técnica do jato de água, com 50 ou $100 \mathrm{ml}$, em determinar a prevalência da infecção por $\boldsymbol{P}$. cuniculi nos condutos auditivos examinados foi utilizado o teste de $\chi 2$. $\mathrm{O}$ teste não paramétrico de Mann e Whitney foi utilizado para comparar o percentual de recuperação e os índices parasitários obtidos mediante a lavagem dos condutos auditivos com $50 \mathrm{~mL}$ ou $100 \mathrm{~mL}$.

$\mathrm{O}$ número de condutos encontrados com ácaros nas lavagens com 50 e 100ML apresentamse na tabela 1. Esses resultados demonstram que a técnica da lavagem do conduto auditivo é eficiente para estudos de prevalência de infecção por $\boldsymbol{P}$. cuniculi, pois conseguiu identificar o parasito em 22 (91\%) dos 24 condutos infectados na lavagem com $100 \mathrm{~mL}$ e em $18(78,3 \%)$ de 23 condutos infectados com a lavagem de com 50mL (Tabela 1). Para os condutos auditivos lavados com $50 \mathrm{~mL}$, a prevalência da infecção, considerando os condutos auditivos encontrados com ácaros após a utilização das duas técnicas (lavagem e abertura do conduto auditivo), foi de $80 \%(24 / 30)$, enquanto que, para os lavados com $100 \mathrm{~mL}$, foi de 76,6\% (23/30), não apresentando diferença estatística significativa $(\mathrm{P}=5,81)$. SANTOS et al. (2006), utilizando vários jatos de água em um dos condutos auditivos, observaram uma prevalência de 73\% na Paraíba, e FACCINI \& RIBEIRO (2008), no Rio de janeiro, lavando um conduto auditivo com $40 \mathrm{~mL}$, encontraram prevalência de $62 \%$.

Em alguns casos, foi identificado um maior número de condutos auditivos positivos através da lavagem do que com a técnica de abertura do conduto (Tabela 1). Isso significa que, na lavagem, foram recuperados todos os ácaros presentes, o que ocorreu, principalmente, naqueles condutos sem crostas e sem cerúmen, nos que predominavam as formas de ovo, larva e ninfa de $\boldsymbol{P}$. cuniculli.

Tabela 1 - Prevalência de infecção por Psoroptes cuniculi em caprinos abatidos, mediante a lavagem e abertura dos condutos auditivos.

\begin{tabular}{|c|c|c|c|c|c|c|}
\hline \multirow[t]{2}{*}{ Parâmetros } & \multicolumn{3}{|c|}{------Em condutos auditivos lavados com 50ml------ } & \multicolumn{3}{|c|}{-------Em condutos auditivos lavados com $100 \mathrm{ml------}$} \\
\hline & Lavagem $^{\text {a }}$ & Abertura $^{\mathrm{b}}$ & Lavagem + abertura $^{\mathrm{c}}$ & Lavagem & Abertura & Lavagem + abertura \\
\hline Condutos examinados & 30 & 30 & 30 & 30 & 30 & 30 \\
\hline Condutos positivos & 22 & 21 & 24 & 18 & 18 & 23 \\
\hline
\end{tabular}

${ }^{\mathrm{a}}$ Número de condutos encontrados com ácaros na lavagem; ${ }^{\mathrm{b}}$ número de condutos encontrados com ácaros na abertura; ${ }^{\mathrm{c}}$ número de condutos encontrados com as duas técnicas. 
Em relação aos índices parasitários, não houve diferença estatística entre os condutos auditivos esquerdos (lavados com 100mL) e direitos (lavados com 50mL). Para os condutos direitos, os valores foram os seguintes: intensidade média de infestação, 3,54 18,3 ; variação da intensidade, 3 a 1047; abundância média, $0,11 \pm 6,5$; e densidade de ácaros no conduto, 2559. Para os condutos esquerdos, os índices foram: intensidade média de infestação,

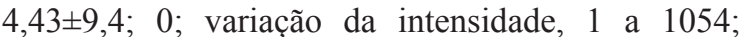
abundância média, $0,14 \pm 0,31$; e densidade de ácaros no conduto, 3392.

Somente três dos 30 caprinos que tiveram os condutos examinados apresentaram sinais clínicos de otocariase, incluindo aumento da quantidade de cerúmen, crostas lameladas e esbranquiçadas e secreção purulenta. Os demais animais não apresentavam sinais clínicos, confirmando assim que a sarna psoróptica na região nordeste é predominantemente subclínica (FACCINI et al., 1992).

Quanto à eficiência da técnica na recuperação dos ácaros, verificou-se um percentual médio de recuperação de $19 \%$ a $35 \%$ para todos os estádios de desenvolvimento, exceto para o estádio de ovo, que foi de $11 \%$ a $15 \%$ (Tabela 2 ), não havendo diferença estatística significativa $(\mathrm{P}>0,05)$ entre as taxas de recuperação com 50 e $100 \mathrm{~mL}$ de água. Para os ovos, a recuperação foi significativamente menor $(\mathrm{P}<0,05)$ do que para os demais estádios. Apesar da técnica do jato de água permitir a comprovação do parasitismo por $\boldsymbol{P}$. cuniculi em animais infestados, para estudos de dinâmica populacional, recomendase a abertura e contagem dos ácaros restantes, já que uma grande quantidade de ácaros não são coletados com a lavagem do conduto auditivo. Os resultados da tabela 2 demonstram que o número de ácaros

Tabela 2 - Percentual médio de recuperação de diferentes estádios biológicos de Psoroptes cuniculi, em caprinos, obtido através da técnica do jato de água com 50 e 100mL.

\begin{tabular}{lcc}
\hline Estádios Biológicos & ---Percentual médio de recuperação--- \\
\hline & $50 \mathrm{~mL}$ & $100 \mathrm{~mL}$ \\
Fêmeas & $25,6 \pm 36,4^{\mathrm{Aa} *}$ & $31,0 \pm 39,9^{\mathrm{Aa}}$ \\
Machos & $29,4 \pm 38,9^{\mathrm{Aa}}$ & $21,2 \pm 40,9^{\mathrm{Aa}}$ \\
Ninfas & $19,4 \pm 36,7^{\mathrm{Aab}}$ & $32,7 \pm 41,3^{\mathrm{Aab}}$ \\
Larvas & $34,6 \pm 39,4^{\mathrm{Aa}}$ & $30,8 \pm 37,8^{\mathrm{Aa}}$ \\
Ovos & $11,25 \pm 26,87^{\mathrm{Ab}}$ & $14,95 \pm 26,67^{\mathrm{Aac}}$ \\
\hline
\end{tabular}

*Médias seguidas de letras maiúsculas na mesma linha não diferem estatisticamente $(\mathrm{P}>0,05)$ e médias seguida de letras minúscula na mesma coluna não diferem estatisticamente $(\mathrm{P}>0,05)$ encontrados na lavagem dos condutos auditivos de caprinos abatidos representa aproximadamente um terço dos parasitas presentes neles. A baixa eficiência de recuperação de ácaros encontrada neste estudo difere da observada por FACCINI et al. (1987) para $\boldsymbol{R}$. auris e pode estar relacionada à diferenças de hábito alimentar entre os ácaros dos gêneros Railletiae e Psoroptes. P. cuniculi se alimenta formando crostas pardacentas, aderentes, sendo os ácaros encontrados em abundância sob as crostas, diferente dos ácaros do gênero Railletia, que se encontram soltos no conduto auditivo (SWEATMAN, 1958)

Conclui-se que a técnica da lavagem do conduto auditivo, usando-se 50 ou $100 \mathrm{ml}$ de água, é eficiente para estimar a prevalência da otocaríase em caprinos, pois identifica entre $78,3 \%$ e $91 \%$ dos condutos infectados. No entanto, para determinar a prevalência real, é necessário realizar a abertura do conduto auditivo para observação dos ácaros. Para quantificar o parasitismo pelo ácaro, recomenda-se a abertura do conduto e a contagem dos ácaros, pois, com a lavagem, recuperam-se aproximadamente um terço dos parasitos.

\section{COMITÊ DE ÉTICA E BIOSSEGURANÇA}

Declaração dos autores: Nós, autores do artigo intitulado "Eficiência da técnica de lavagem do conduto auditivo para medir a infestação por Psoroptes cuniculi em caprinos", declaramos, para os devidos fins, que o projeto que deu origem aos dados deste não foi submetido para avaliação ao Comitê de Ética da Universidade Federal de Campina Grande, mas estamos cientes do conteúdo das resoluções do Conselho Nacional de Controle de Experimentação Animal-CONCEA “htp://www.mct.gov.br/index. php/contente/view/310553.html" caso envolva animais. Dessa forma, os autores assumem total responsabilidade pelos dados apresentados e estão disponíveis para possíveis questionamentos, caso venham a ser requeridos pelos órgãos competentes.

\section{REFERÊNCIAS}

ARAÚJO, T.L Sobre a ocorrência no Brasil da sarna psoróptica dos caprinos e sarna notoédrica do gato doméstico. Revista da Faculdade de Veterinária da Universidade de São Paulo, v.2, n.1, p.24-32, 1941.

COSTA, C.A.F; VIEIRA, L.S. Ectoparasitos permanentes em caprinos e ovinos em Sobral, CE. Pesquisa Agropecuária Brasileira, v.19, n.5, p.639-646, 1984.

FACCINI, J.L.H. et al. Otocaríase psoróptica dos caprinos. Infestação subclínica. Pesquisa Agropecuaria Brasileira, v.16, n.5, p.725-726, 1981.

FACCINI, J.L.H. et al. Evaluation of and ear flushing technique as a post-mortem measure of infestation of Railletia auris. Experimental Applied Acarology, v.3, p.175-178, 1987. Disponível em: <http://link.springer.com/article/10.1007/BF0127 0479\#page-1>. Acesso em: 27 abr. 2007. 
FACCINI, J.LH; COSTA, A.L. Subclinical psoroptic otocariasisin Brazilian sheep with comments on a technique for mite collection. Experimental \& Applied Acarology, v.13, p.227-229, 1992. Disponível em: <http://link.springer.com/article/10.1007/BF01194 938\#page-1>. Acesso em: 25 mar. 2012.

FACCINI, J.LH; RIBEIRO, V. Raillietia caprae (acari: raillietidae) and Psoroptes ovis (Acari: Psoroptidae) in the ears of goats in the state of Rio de Janeiro, Southeast Brazil. Revista Brasileira de Parasitologia Veterinária, v.17, p.59-61, 2008. Disponível em: $<$ http://www.scielo.br/scielo.php?pid=S1984-2961200800010 0014\&script $=$ sci_arttext $>$. Acesso em: 26 mar. 2013. doi: 10.1590/ S1984-29612008000100014.

LEITE, R.C. et al. Avaliação de uma técnica in vivo para medir a infestação por ácaros do gênero Raillietia Troussart (Acari) em bovinos. Memorias do Instituto Oswaldo Cruz, v.84, supl.IV, p.309-311, 1989
MARGOLIS, L. et al. The use of ecological terms in parasitology. Journal of Parasitology, v.68, n.1, p.131-133, 1982. Disponível em: <http://www.jstor.org/discover/10.2307/3281335?uid=3737 $664 \&$ uid $=2 \&$ uid $=4 \&$ sid $=21102915377523>$. Acesso em: 27 jan. 2013.

NUNES, V.A.; NUNES, I.J. Técnica de exame post mortem do sistema auditivo aplicada ao estudo de otites em bovinos. Arquivos da Escola de Veterinária da UFMG, v.27, p.155-161,1975.

SANTOS, A.C.G. et al. Artrópodes parasitos de caprinos do Sertão Paraibano. Agropecuária Científica no Semi-árido, v.2, n.1, p.917, 2006. Disponível em: <http://150.165.111.246/ojs-patos/index. php/ACSA/article/viewFile/15/pdf>. Acesso em: 14 jul. 2010.

SWEATMAN, G.K. On the life history and valididy of the species in Psoroptes, a genus of mange mites. Canadian Journal of Zoology, v.36, p.905-29, 1958. 